\title{
Théologie et sciences des religions : hier et aujourd'hui
}

Jean Séguy

\section{(2) OpenEdition}

1 Journals

Édition électronique

URL : http://journals.openedition.org/assr/176

DOI : $10.4000 /$ assr. 176

ISSN : $1777-5825$

Éditeur

Éditions de l'EHESS

Édition imprimée

Date de publication : 1 avril 2002

Pagination : 21-28

ISBN : 2-222-96718-X

ISSN : 0335-5985

\section{Référence électronique}

Jean Séguy, "Théologie et sciences des religions : hier et aujourd'hui », Archives de sciences sociales des religions [En ligne], 118 | avril - juin 2002, mis en ligne le 14 novembre 2005, consulté le 01 mai 2019. URL : http://journals.openedition.org/assr/176 ; DOI : 10.4000/assr.176 


\section{THÉOLOGIE ET SCIENCES DES RELIGIONS : HIER ET AUJOURD'HUI}

"Il y a un moment pour tout et un temps pour toutes choses sous le ciel » Qo.3,1.

À propos de :

GISEL (Pierre), La Théologie face aux sciences religieuses ; différences et interactions, Genève, Labor et Fides, 1999, 299 p. (bibliogr., index des noms).

KIPPENBERG (Hans-Gerhard), À la découverte de l'histoire des religions. Les sciences religieuses et la modernité, Paris, Salvator, 1999, 423 p. (Préface de Jean Greisch) (bibliogr., index des noms, index des lieux).

MOLENDIJK (Arie L.), PELS (Peter), eds. Religion in the Making. The Emergence of the Sciences of Religion. Leyde, Boston, Cologne, E.J. Brill, 1998, XII-318 p. (bibliogr., index des thèmes, index des noms).

Recherches de Science Religieuse, $\mathrm{n}^{\mathrm{O}} 1$ (janv.-mars 2000), t. 88, 1, Dossier : "L'expérience de la Vérité », pp. 1-164 et $\mathrm{n}^{\mathrm{o}} 2$ (avr.-juin 2000), t. 88, 2, Dossier : "Science des religions ou théologie ? Ernst Troeltsch aujourd'hui », pp. 165-321.

Les rapports entre sociologie et religion (théologie éventuellement) ne vont pas constamment dans une seule direction : ils étaient souvent tendus lorsque les Archives commencèrent d'exister (1956) et le restèrent longtemps ${ }^{1}$. Les trois ouvrages et les deux livraisons de revue commentés dans la présente Note suggèrent une autre situation actuelle - plus complexe - de ces rapports. L'événement vaut d'être signalé.

$$
\text { *** }
$$

${ }^{1}$ Jean SÉGuY, «Panorama des sciences des religions », in Henri Desroche et Jean SÉGuY, éds., Introduction aux sciences humaines des religions, Paris, Cujas, 1970, pp. 35-72. 
Les trois ouvrages ici évoqués (P. Gisel, H.-G. Kippenberg, A.L. Molendijk et Pels) ont en commun un même projet : s'interroger sur les sciences religieuses dans leurs rapports - au passé mais aussi au présent - avec les religions qu'elles étudient, leurs théologies et leurs théologiens en particulier. Pourtant aucun de ces ouvrages ne ressemble à son voisin.

Professeur de théologie à la Faculté (protestante) de théologie de l'Université de Lausanne, P. Gisel, auteur de La Théologie face aux sciences religieuses, a été récemment le maître d'œuvre d'une notable Encyclopédie du protestantisme (Labor et Fides, 1995); il est connu aussi comme interprète et diffuseur - en domaine francophone - de l'œuvre d'Ernst Troeltsch. C'est en tant que théologien « postlibéral »- il se qualifie ainsi - que P. Gisel s'intéresse, dans l'ouvrage plus haut nommé, aux problèmes d'une interaction positive possible entre théologie et sciences des religions. Dans la perspective qui est la sienne, fortement inspirée et de Max Weber et d'Ernst Troeltsch, théologie et sciences des religions - sociologie historique et comparative en premier lieu - coopèrent de façon interactive et complémentaire. On ne saurait mieux affirmer, dès le point de départ, qu'entre les diverses approches possibles des phénomènes religieux, aucune concurrence ni aucune hiérarchisation n'apparaît recevable. Gisel tient pour évident que théologie, histoire, sociologie, psychologie des phénomènes religieux coexistent comme autant de spécialités promptes au comparatisme et à la pluridisciplinarité, dans une entière autonomie de la démarche intellectuelle. L'ouvrage de P. Gisel propose en conséquence que "la théologie redéfinisse sa tâche, voire change de paradigme ». C'est bien d'une « réforme de la théologie » qu'il s'agit là, réforme qui renoncerait - en cas de passage du «pas encore » au «déjà » - à toute légitimité confessionnelle.

Il y a trois parties dans cette Théologie face aux sciences religieuses. On peut les lire, croyons-nous, comme autant d'exercices pratiques offerts en illustration du programme immédiatement et rapidement esquissé. La première partie étudie la tâche de la théologie dans la crise religieuse actuelle, en même temps que dans la crise de l'Occident et de la modernité. Elle s'y livre autour des thèmes de l'institutionnalisation de la religion et des recompositions religieuses en cours. La deuxième partie met en dialogue le dossier de la "mystique » aujourd'hui avec celui des rapports entre christianisme et modernité ; par ailleurs elle se penche sur l'épineux problème d'un «horizon universel quant à la compréhension du religieux $»^{2}$. Enfin la troisième partie revisite les « questions ouvertes » de l' « absoluité » du christianisme et de sa place dans une "théorie de la religion ».

Derrière tout le déploiement de science et la passion du vrai qui anime l'auteur, on distingue sans mal des influences diverses: elles vont de Schleiermacher au néo-kantisme, à Dilthey, Max Weber, Georg Simmel, Troeltsch (ombre tutélaire et « dépassée »), à bien d'autres encore - dont celle de Ritschl. Mais ce qui étonnera encore plus le lecteur, c'est de constater la connaissance que démontre Gisel en matière d'approches non-théologiques contemporaines des religions. On ne s'étonnera donc pas qu'il partage avec certains auteurs récents deux convictions de plus en plus répandues parmi les sociologues actuels - y compris dans le milieu des Archives -, à savoir que modernité et religion ne s'excluent pas nécessairement,

${ }^{2}$ Sur ce problème et à propos de la mystique et du bouddhisme, GisEl (op. cit., pp. 95-99) se différencie clairement de Eugen DrEWERMANN. 
d'une part; que, d'autre part, la modernité elle-même se révèle capable de «produire du religieux ». Notons encore que, pour P. Gisel, la mystique (ou le spiritualisme) - au sens où Troeltsch entend ces choses - profite actuellement de cette capacité du moderne à générer du religieux; mais cela ne saurait permettre l'auteur le signale à plusieurs reprises - au spiritualisme-mystique contemporain d'échapper aux problèmes de la légitimation et de l'institutionnalisation.

$$
* *
$$

L'ouvrage de H. G. Kippenberg est, lui, consacré à La découverte de l'Histoire des religions. Mais le sous-titre nous avertit que l' " histoire des religions » est ici considérée comme l'une des "sciences des religions " dans le rapport de ces approches au problème englobant de la modernité ${ }^{3}$. Professeur d'histoire des religions à l'Université de Brême (Allemagne), l'auteur n'hésite pas à parler de ruptures et de changements. Il s'agit pour lui en effet de signaler « le rôle capital qu'ont joué les thèmes religieux dans la formation de l'identité moderne ». La séparation aujourd'hui tenue pour constitutive entre Églises, théologie et sciences religieuses fut l'œuvre des Lumières. Dès lors les discours divergents ont foisonné autour de la religion, trouvant leur point d'équilibre dans la dénonciation de l'aliénation et de l'obscurantisme religieux. Il semble cependant que la multiplication et l'extension des recherches sur les peuples «primitifs », l'Antiquité et les autres époques historiques, en des pays variés, et sur des formes diverses de religion ou des religions variées aient généralement permis de revenir sur les préjugés rapportés plus haut. De nos jours en tout cas, et selon Kippenberg, les spécialistes des sciences des religions semblent - pour la plupart - considérer la religion comme une donnée anthropologique constitutive de l'humain. Dès lors on ne s'étonne plus qu'il existe une histoire religieuse des sociétés et des individus modernes. Kippenberg s'intéresse beaucoup à l'anthropologie, à la sociologie aussi (Durkheim sans doute, mais Weber en tout particulier), à la psychologie religieuse - au moins en ses débuts, autour de l'œuvre de W. James singulièrement. Mais on voit Kippenberg attiré encore par R. Otto, N. Söderblom et van der Leeuw. On remarque aussi que cette «Découverte de l'Histoire des religions » lie fortement les évolutions de la philosophie, de la théologie - chrétienne essentiellement et protestante en tout particulier -, de l'histoire au sens moderne de la chose et de la philosophie de l'histoire (pour autant que l'une et l'autre aillent ensemble) dans l'émergence progressive des sciences des religions; elle porte attention aussi aux évolutions dans l'étude des langues - anciennes en particulier - tout au cours du XIX ${ }^{\mathrm{e}}$ siècle spécialement. C'est, selon notre auteur, entre 1850 et 1920, dans l'interaction de cet ensemble de

\footnotetext{
${ }^{3}$ Une ambiguïté court au travers de tous les textes ici commentés : y parle-t-on de diverses sciences des religions? ou de la religion? d'une science de la religion (ou encore des religions) ? On n'a pas l'impression que les auteurs distinguent entre ces diverses formulations possibles et que, lorsqu'ils le font, ils en tirent des conséquences théoriques et pratiques. Souvent, et de plus, on a le sentiment que pour certains histoire des religions et science de la religion s'équivalent, quoi qu'il en soit du contenu prêté à ces termes ! De même, il est partout question de modernité dans les textes faisant l'objet de la présente Note, mais sans que la nature de la chose soit précisée outre mesure. Certains de nos auteurs ne manquent pas d'ailleurs de soupçonner qu'histoire, philosophie et science n'entretiennent parfois quelques rapports (tout entier encore à mesurer) lorsqu' " on » parle de religion et même des religions !
} 
« facteurs » immédiatement désignés ${ }^{4}$ que naissent peu à peu et se développent les approches modernes et contemporaines des cultures, et les sciences des religions comme études particularisées des cultures religieuses, ou des religions comme éléments constituants de toute culture.

L'ouvrage de Kippenberg est - en traduction française - précédé d'une Préface assez longue (pp. 1-19) signée par Jean Greisch, professeur de philosophie à l'Institut Catholique de Paris. On ne manquera pas de la lire, car elle constitue un résumé admirable - selon nous et dans sa fidélité au texte de l'auteur - de l'ensemble du livre. La « thèse conclusive » du Professeur de Brême est ainsi rapportée par le préfacier : ... «la vie des hommes dans les sociétés modernes ne cessera d'avoir besoin d'un minimum de continuité avec les religions. À cela s'ajoute la thèse non moins importante qu'en inscrivant le processus de la modernisation dans l'histoire des religions, les sciences religieuses ont directement contribué à restituer aux religions un 'droit de séjour' dans la culture moderne ». Comparant cette conclusion avec celle du Désenchantement du monde - de Marcel Gauchet (Gallimard, 1985), Jean Greisch se demande si les perspectives de Hans-Gerhard Kippenberg peuvent recueillir l'adhésion de la plupart des lecteurs de son livre. Il en doute, ne serait-ce que parce que la façon d'écrire l'histoire des religions varie avec les pays. Sans doute ; il est probable de plus - croyons-nous - qu'en un même pays où au sein de traditions idéologiques, religieuses, politiques (et autres) diverses, l'histoire - celle des religions y comprise - est susceptible de variations encore plus nombreuses. Il reste que - on le vérifiera dans la présente Note - des chercheurs contemporains œuvrant sur des prémisses autres pensent eux aussi (c'est le cas de P. Gisel dont on a présenté les perspectives plus haut) qu'il y a bien un avenir pour la (ou les) religion(-s) dans la modernité.

$$
* * *
$$

Cette conviction que nous venons de dire se trouve encore dans l'ouvrage collectif sous la double direction d'une part, d'A. L. Molendijk, théologien et historien des théologiens et des théologies (spécialiste d'E. Troeltsch et de son œuvre composite), et d'autre part, de P. Pels habitué de l'anthropologie culturelle et de l'étude des phénomènes religieux dans les pays du Tiers-Monde. Comme beaucoup de collectifs, Religion in the Making offre à lire les Actes d'un Colloque. Celui dont est issu le présent ouvrage s'est déroulé au Centre de Recherches «Religion et société » de l'Université d'Amsterdam, entre le 22 et le 24 mai 1997. L'anglais semble avoir été la langue de travail de cette réunion dont les douze communicants provenaient d'Europe du Nord (Pays-Bas, pays scandinaves, Grande-Bretagne, Allemagne) et d'Amérique du Nord, Canada compris. L'Introduction de ces Actes (pp. 1-27) est due à A. L. Molendijk. On y trouve une véritable et savante mise au point de nos connaissances sur les débuts, entre 1850 et 1920, des sciences des religions dans leur variété. Pour autant, le choix des limites chronologiques n'est pas expliqué. On en est réduit à l'hypothèse - raisonnable selon nous - que les sciences

\footnotetext{
${ }^{4}$ On ne saurait nier l'importance, dans la naissance des sciences des religions, des « facteurs idéels », en particulier de l'influence d'un auteur - ou d'un livre - sur un autre ; est-ce une raison suffisante pour ne pas prêter attention aux facteurs matériels dans le processus global de cette genèse confirmée ? Il faut saluer ici la tentative en ce sens faite par MoLENDIJK dans l'introduction à l'ouvrage sous sa codirection, autour des premiers phénomènes d'institutionnalisation.
} 
des religions naissent entre l'échec de la révolution de 1848 et la mort de Max Weber. Si elle se révèle exacte, cette hypothèse apparaît pleine de sens...; mais quelques lignes d'explication à ce sujet auraient remplacé avantageusement selon nous les considérations générales qui ouvrent cette Introduction (pp. 1-11) et concernent la science ut sic dans son idéal moderne. La deuxième partie des réflexions de Molendijk se consacre - rapidement - à l'«historiographie » des sciences des religions et souligne l'importance des " signes d'institutionnalisation » dans la première histoire de ces « approches » : création de chaires, de revues spécialisées, de conférences publiques, de congrès et de colloques de spécialistes, etc. Enfin la troisième partie de la même Introduction présente en des analyses très précises les douze communications qui forment le plus gros du livre. Les lecteurs des Archives ne manqueront pas de relever, parmi ces travaux celui - il ouvre le recueil de Michel Despland intitulé «Sciences of Religion in France During the July Monarchy (1830-1848) »: il y apparaît que les sciences des religions se pratiquaient en France avant toute institutionnalisation et avant 1850.

On lira aussi avec intérêt l'amusante et érudite communication de Ivan Strenski ayant pour titre «The ironies of Fin de Siècle Rebellions Against Historicism and Empiricism in the EPHE Fith Section»; on y voit l' « historicisme » l'emporter de façon incertaine - en fin XIX ${ }^{\mathrm{e}}$ siècle - sur l' « empirisme théorique » des sociologues !

En ce qui nous concerne plus directement dans la présente Note, on relèvera que certaines communications font allusion à la diversité des attitudes des clergés devant l'émergence - en face des théologies - des sciences des religions. On note en particulier que beaucoup des premiers porteurs de ces approches "modernes » furent - en pays protestants - des pasteurs. Le bon goût et le fonctionnement ordinaire de la mémoire savante auraient demandé, croyons-nous, que soit rappelé à cette occasion le sort malheureux des clercs catholiques - historiens et exégètes principalement - qui choisirent le camp des "sciences des religions ». Le modernisme, " hérésie moderne » s'il en fut, naquit en effet de l'attachement manifesté par certains d'entre eux aux méthodes et aux perspectives problématiques de la Religionsgeschichtliche Schule. Le rappel de cette information banale nous permet, en tout cas et ici, de passer sans efforts d'un colloque de savants nord-européens et nord-américains à la revue évoquée aux quatrième et cinquième rangs des items motivant, à l'initiale, la présente Note.

$$
\text { *** }
$$

La revue trimestrielle Recherches de Science Religieuse, qui fournira l'occasion des réflexions qui suivent, est née en $1910^{5}$ dans le cadre des publications périodiques de la Compagnie de Jésus en France. Il s'agissait, en la créant, de décharger les Études, revue jésuite de culture générale fondée en 1854, du souci des problèmes théologiques que l'époque, celle du modernisme ${ }^{6}$, tendait à multi-

${ }^{5}$ Le modernisme fut condamné par le magistère catholique en trois documents successifs : l'encyclique Providentissimus (1893, Léon XIII), le décret du Saint-Office «Lamentabili sane exitu » et enfin l'encyclique Pascendi dominici gregis (8 septembre 1907, Pie X). Sur les circonstances de la naissance des Recherches, voir Pierre VAllin, Histoire d'une revue; une aventure jésuite ; des origines au Concile Vatican II (1856 à 1965), numéro spécial d’Études, avril 2000, p. 13 et pp. 23-27.

${ }^{6}$ Émile Poulat, Histoire, dogme et critique dans la crise moderniste, Paris, Casterman, 1962 (« $3^{\text {e }}$ éd., suivie de 'La réflexion d'Alphonse Dupront'», Paris, Albin Michel, 1996). 
plier. On a pu considérer les Recherches, que dirigeait le P. Léonce de Grandmaison, théologien et spirituel jésuite d'envergure, comme un instrument du combat contre ledit modernisme ${ }^{7}$. Elles le furent sans doute, mais non sans discernement; ne serait-ce qu'à travers leur ouverture - et celle de leur directeur - aux problèmes familiers à plus d'un moderniste, de la mystique comparée ${ }^{8}$. Ce n'est pas un hasard si ce milieu des Recherches produisit - sous la direction du P. Joseph Huby, le collectif Christus, manuel d'histoire des religions (Paris, Beauchesne, $1912 ; 6^{\mathrm{e}}$ éd. en 1934) ${ }^{9}$. C'est encore de cette initiative - devenue ancienne - que se réclame expressément, cinquante-trois ans plus tard, la publication de La mystique et les mystiques (Paris, Desclée De Brouwer, 1965), sous la direction d'André Ravier et toujours dans le même milieu des Recherches. Le P. Henri de Lubac - pas encore cardinal alors - préfaça longuement cette Mystique et les mystiques qui se réclame expressément des sciences religieuses ${ }^{10}$.

Or, il convient de le remarquer, c'est le même milieu qui tourne aujourd'hui son attention vers les «Sciences des religions" dans leur rapport à la théologie. Cela ressort avec clarté du titre - interrogatif néanmoins - donné à la livraison d'avril-juin 2000 des Recherches : «Sciences des religions ou théologie ? Ernst Troeltsch aujourd'hui ». On lira sous ce titre quatre communications issues d'un colloque tenu à Paris et au Centre Sèvres (Facultés jésuites de philosophie et de théologie) le 27 janvier $1999^{11}$. L'occasion de cette réunion où se rencontraient des

${ }^{7}$ Pierre VALlin, « Grandmaison, Léonce Loyzeau de, 1868-1927 » in François LaPLANChe, éd., Dictionnaire du monde religieux, t. 9, Les sciences religieuses, le XIX $X^{e}$ siècle, 1800-1914, Paris, Beauchesne, 1996, pp. 294-296. On étudiera la distance (proximité et/ou éloignement) entre les textes scientifiques et les interventions du spirituel, en se penchant sur le texte et les notes de La Religion personnelle, Paris, Gabalda, 1927 (le tirage de 1944 s'annonce comme « onzième mille »).

${ }^{8}$ Émile Poulat, Critique et mystique. Autour de Loisy, ou de la conscience catholique et l'esprit moderne, Paris, Le Centurion, 1984. Aussi, du même et plus récent, L'Université devant la mystique. Expérience de Dieu sans mode. Transcendance du Dieu d'Amour, Paris, Salvator, 1999 (cf. Arch. 112.96).

${ }^{9}$ Pierre Vallin, «Huby, Joseph, 1878-1948 » in François Laplanche, éd., op. cit., pp. 332-333.

10 On se permet de renvoyer ici à notre recension de La Mystique et les mystiques dans Archives de sociologie des religions, 22, juill.-déc. 1966, n 217 (pp. 215-216). De plus, on se plaît à souligner l'importance de la Préface que le P. Henri de Lubac écrivit pour cet ouvrage. Il y jalonne en effet, de façon informée et intéressante, le parcours accompli en matière de science de la religion (ou de sciences religieuses, ou encore d' «histoire des religions ») par l'intelligentsia catholique, entre les premiers débats autour du modernisme et la période contemporaine de cette Préface même : et, pour le dire de deux noms ici cités expressément, de Salomon ReINAch (Orpheus, Paris, Alcide Picard, 1909, 627 p. ; nombreuses rééditions), à Michel DE CERTEAU (en 1965, à la parution de La Mystique, etc., il s'est déjà fait un nom dans 1'histoire des spirituels (P. Favre, J.-J. Surin, Teilhard de Chardin...). Mais la distance reste à évaluer qui va de ce dernier nommé aux propositions de GiSEL (op. cit., pp. 27-34; 43-51) ou à celles que livrent les $n^{\circ} 88 / 1$ et 2 des Recherches.

${ }^{11}$ Voici les titres des communications en question : Joseph MoIngt (Centre Sèvres, Paris) : « Ernst Troeltsch, Science des religions ou théologie ? »; Hartmut RuDDIES (Saint-Pétersbourg), " Culte christique et exigence communautaire; la conception christologique d'Ernst Troeltsch "; Camille FroIDEVAUX (EHESS), «Lire Ernst Troeltsch en France aujourd'hui »; Jean-Marc TÉTAZ (Kulturwissenschaftliches Institut, Essen), «Rédemption, eschatologie et sublimation : éléments pour une théorie du christianisme : Ernst Troeltsch et Max Weber ». Ce dernier article (de J.-M. TÉTAZ) mérite lecture de quiconque s'intéresse aux rapports intellectuels entre Weber, Simmel et Troeltsch. On doit ajouter que P. GiseL signe l'«Avant-Propos » (pp. 169-177) présentant l'ensemble de ces cinq communications ci-dessus. Il souligne ce faisant que la question posée en l'occurrence porte essentiellement sur « le statut de la théologie en modernité, aussi vrai qu'Ernst Troeltsch entend en reprendre ou en poursuivre la tâche dans le cadre d'une «science générale de la religion » (ibid., p. 169). De la « relecture de Troeltsch » impliquée en l'occurrence on trouvera trace également, au moins sous la forme d'un " esprit», dans le $\mathrm{n}^{\circ} 88 / 1$ des Recherches (janv.-mars 2000) dont le dossier principal porte sur «L'expérience de la vérité » 
théologiens catholiques et la Faculté de théologie protestante de Lausanne ainsi que des chercheurs présentés comme "indépendants » était procurée par la parution de Histoire des religions et destin de la théologie, titre donné au volume III des Euvres d'Ernst Troeltsch (traduites en français au Cerf (Paris) et chez Labor et Fides (Genève) (cf. Arch. 104.125). Si on y ajoute que P. Gisel fut à l'initiative de la traduction française des Euvres de Troeltsch et du colloque de janvier 1999, on peut dire que la présente Note de lecture se conclut là où elle a commencé.

$$
\text { *** }
$$

Nous nous demandions dans notre Introduction au présent essai ce qu'il en était des rapports actuels entre sociologie - ou sciences des religions plus généralement parlant - et théologie - entre autres champs possibles du domaine religieux. Pouvait-on les décrire en se contentant d'une opposition entre absence ou présence de tensions entre l'un et l'autre pôles observés?

La réponse à notre question, telle qu'on peut la lire dans les ouvrages plus haut commentés, peut se dire à partir du livre de P. Gisel signalé en premier lieu. Selon nous, elle s'y exprime de deux façons: comme conviction et comme exercice. Comme conviction: selon nos auteurs, dont Gisel apparaît ici comme le porte-parole de fait, les rapports entre théologie et sciences des religions ne doivent pas s'exprimer nécessairement en termes de tensions ou d'absence de tensions entre elles, c'est-à-dire en perspective de conflit ; la possibilité ne saurait être exclue $a$ priori que dans la pratique, et jusqu'à un certain point, théologie et sciences religieuses puissent se montrer complémentaires l'une de l'autre. L'ouvrage de Gisel (La théologie face aux sciences religieuses) constitue précisément l'exercice démontrant concrètement la praticabilité de la conviction précédente. On pourrait sans doute avoir bien des questions à poser aux tenants de cette perspective. Mais il n'importe ici : il suffit à cette Note d'avoir pu signaler deux choses : 1) l'existence d'un courant actuel parmi les théologiens et chez les pratiquants des sciences non théologiques des religions qui voit un avenir pour les religions dans la modernité ; 2) l'existence d'un courant théologique complémentaire du précédent pour qui le recours aux sciences des religions vise expressément à une "réforme de la théologie » et à un « régime nouveau de sa pratique en christianisme ». Selon nous, c'est à étudier et illustrer certains aspects de cette utopie déjà quasiment pratiquée que s'emploient certains des participants au Colloque Troeltsch de 1999 et au no 2 tome 8 des Recherches. On rejoint ici certains aspects contemporains jugés positifs des rapports entre sciences des religions et théologie chrétienne signalés par certains auteurs dans l'ouvrage collectif d'hommage à Karel Dobbelaere recensé il y a peu, et par nos soins, dans une revue anglaise de notre spécialité ${ }^{12}$. Selon ces auteurs et dans le commentaire que nous en faisions, on peut affirmer en effet ceci : à côté de tensions persistantes entre théologie et sciences sociales des religions, il existe aujourd'hui - aussi et de plus en plus, en certains pays en tout cas - des aires de recherches où la collaboration s'affiche sans réticences entre approches théologiques et non-théologiques des phénomènes religieux. Le cas le plus visible en

12 Jean SÉGUY, compte rendu de Liliane Voyé et Jack Billiet, éds., Sociology and Religions: An Ambiguous Relationship/Sociologie et religions : des relations ambigües, Louvain, Leuven University Press, 1999, Journal of Contemporary Religion, 15/3, oct. 2000, pp. 413-415 (cf. Arch. 114.46). 
l'occurrence est celui des travaux de nature sociologique concernant les Écritures et la première histoire des Églises chrétiennes; deux livraisons de Social Compass, l'une et l'autre sous la direction de P.-A. Turcotte, ont été consacrées à ce phénomène ${ }^{13}$. Tel article de Jean Remy dans le même Hommage à K. Dobbelaere insiste également - et à juste titre - sur le fait que bien d'autres recherches que celles sur l'Église ancienne et la sociologie des Écritures sont menées, fort pacifiquement et sur des sujets religieux, dans le cadre d'universités et de centres de recherche catholiques ou protestants, en Occident surtout ${ }^{14}$.

Toujours dans les mêmes perspectives - et bien que nous n'y ayons pas insisté outre mesure - la présente Note de lecture permet de saisir - c'est particulièrement visible dans le livre de P. Gisel - que le Type mystique de Troeltsch - souvent très critiqué jusqu'ici - démontre pourtant aujourd'hui encore son utilité en plusieurs lieux de l'actualité religieuse ; de même la plupart des théologiens qui viennent de nos jours au contact de l'œuvre du même Troeltsch semblent trouver un intérêt nouveau aux réflexions de cet auteur sur modernité et théologie ${ }^{15}$. Par ailleurs et encore, les trois premiers items commentés plus haut ont permis de relever un ton relativement nouveau dans des travaux d'histoire concernant les spécialités non-théologiques des religions. Il en ressort assez clairement - et si nous les avons bien lus - que les rapports entre les approches non-théologiques et la théologie ont été - dès leurs débuts - au XVIII ${ }^{\mathrm{e}}$ et au XIX ${ }^{\mathrm{e}}$ siècles - très divers : selon les pays, les conjonctures socio-historiques et scientifiques, selon - aussi - les individus, les groupes en présence et la diversité de leurs stratégies réciproques. On peut donc confirmer et généraliser, en Conclusion de la présente Note, les remarques rapides qui en dessinent plus haut l'entrée en matière : les rapports entre sociologie (ou « sciences des religions ») et religion (et/ou théologie) sont des rapports historiques, c'est-à-dire soumis au changement dans l'inattendu de ses variations. En tenir compte ne saurait nuire à la recherche, même la plus contemporaine.

\section{Jean SÉGUY \\ Directeur de Recherche Honoraire CNRS}

\footnotetext{
${ }^{13}$ Voir Social Compass, 39/2, 1992 et 46/4, 1999.

${ }^{14}$ Voir Jean Remy, «L’Église catholique et la sociologie », in Liliane Voyé et Jack BILliEt, op. cit., pp. 101-115.

15 Alors que les Soziallehren, dans lesquels Troeltsch s'exprime assez longuement sur ces problèmes (cf. la Conclusion en particulier) datent de 1912, et l'Absolutheit de 1902.
} 